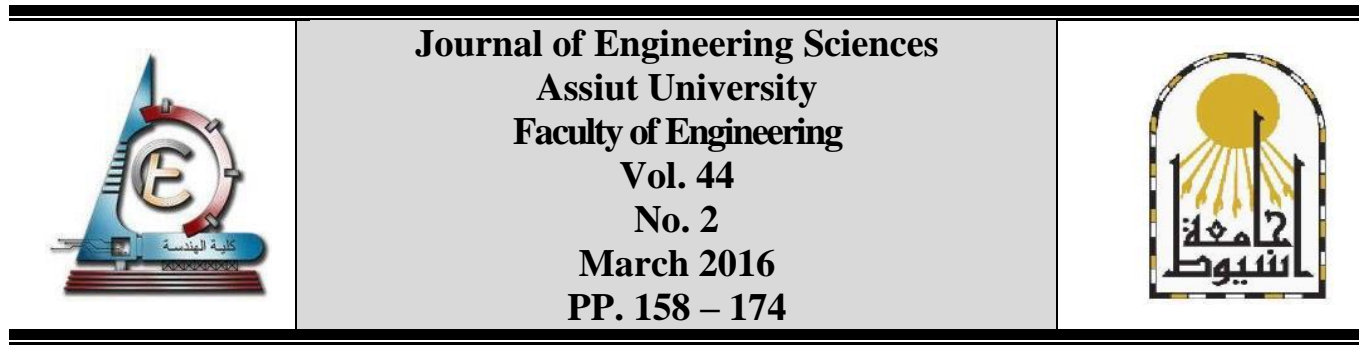

\title{
A DEDICATED DIAGNOSTIC DISTORTION MEASURE FOR THE EVALUATION OF ECG SIGNAL COMPRESSION TECHNIQUES ADOPTED FOR REMOTE DIAGNOSIS OF CARDIOVASCULAR DISEASES
}

\author{
Mohammed M. Abo-Zahhad ${ }^{1,}$, Aziza I. Hussein ${ }^{2}$, \\ Abdelfatah M. Mohamed ${ }^{3}$ and Ahmed M. El-Sawy ${ }^{4}$ \\ ${ }^{1}$ Engineer at Assiut University, Assiut, Egypt \\ ${ }^{2}$ Computer and Systems Department, Faculty of Engineering, Minia University, Minia, Egypt \\ ${ }^{3}$ Electrical and Electronics Eng. Depat., Faculty of Engineering, Assiut University, Assiut, Egypt \\ and in a secondment to Electrical and Computer Eng. Dept., Effat University \\ ${ }^{4}$ Electrical Engineering Department, Faculty of Engineering, Minia University, Minia, Egypt
}

Received 23 February 2016; Accepted 1 March 2016

\begin{abstract}
In the current century diseases of the heart, brain and blood vessels, termed Cardiovascular Diseases (CVDs), represent the main death reasons [1-2]. The CVDs can be diagnosed through the usage of the features extracted from the P-waves, PR-intervals, QRS-complexes, ST-segments, T- and U-waves. The irregularity of beat phases is generally called arrhythmia and some arrhythmias are very dangerous for patients. The types of arrhythmias that can be diagnosed using the Electrocardiogram (ECG) features include: sinus bradycardia, ventricular tachycardia, sinus arrhythmia, atrial premature contraction. In addition, right and left bundle branch block, atrial fibrillation and flutter, heart block as well as coronary artery diseases such as ST- and non ST-elevation myocardial infarction can be diagnosed using ECG signal features.

This paper introduces automatic ECG signal interpretations adopted for detecting CVDs. It investigates the effect of compressing the ECG signals on evaluating the performance of diagnosing CVDs. This has been performed by introducing a Dedicated Diagnostic Distortion Measure (3DM) that is based on comparing the complex features of the original and the reconstructed ECG signals while keeping a pre-determined diagnostic error and good reconstructed signal quality. In fact, the allowable pre-determined diagnostic error is a disease dependent. The diagnostic features considered in this paper include duration features, amplitude features and shape features. Consultations with cardiologists through Mean Opinion Score (MOS) test show that the proposed 3DM is most informative compared to the well-known percentage root-mean square difference in the evaluation of compression
\end{abstract}

\footnotetext{
* Corresponding author.

E- mail address: eng_mmz_eg@yahoo.com
} 
techniques oriented for CVDs diagnosing. The price paid for the usage of 3DM as a good performance metric is the computational complexity in calculating the diagnostic features. However, the calculated features can be adopted for diagnosing purposes.

Keywords: ECG compression; Telediagnoses and Telemonitoring; Cardiovascular disease; Arrhythmias; Blood vessels; MOS test.

\section{Introduction}

The occurrence of heart diseases, mainly depends upon unhealthy habits such as smoking, alcoholism and eating unhealthy foods [3-4]. Several CVDs are due to valvular affection as: mitral valve stenosis, mitral valve regurgitation, aortic valve stenosis, aortic valve regurgitation, and tricuspid valve stenosis, tricuspid valve regurgitation, or ischemic heart diseases as heart attack. These diseases result from the abnormal structure of the heart or its coronary blood vessels. ECG is the most commonly recorded signal in the patient monitoring and diagnosing for quickly detecting cardiac diseases from ECG analysis. The main aim of healthcare management is to develop applications that better identify and track chronic disease states of high risk patients [5-6]. Table (1) includes two categories of risk factors for heart diseases [7].

\section{Table 1.}

Risk factors for heart disease

\begin{tabular}{|l|l|}
\hline \multicolumn{1}{|c|}{ Risk factors that can't be controlled } & \multicolumn{1}{c|}{ Risk factors something can be done about } \\
\hline - Age & - High blood pressure \\
- Gender & - High blood cholesterol \\
- Family history & - Diabetes and overweight \\
- Ethnicity & - Excessive alcohol \\
- Personal history of heart disease & - Physical inactivity \\
& - Smoking and stress \\
\hline
\end{tabular}

Compression of ECG signal is often required for remote CVDs diagnosing to solve the problems of the required huge storage space and the limited bandwidth of the communication channels. In technical literature there exist two types of ECG compression techniques; namely lossless and lossy techniques [8]. In lossless compression techniques, the original signal should be exactly reconstructed while the lossy techniques always involve a loss of information. Lossless methods can only provide limited compression ratios and this storage limitation of lossless techniques made lossy compression of ECG signal an important research topic in biomedical applications. In addition to these, there are many other advantages of lossy ECG compression such as the enhancement of the transmission speed of real-time signals [6], [9]. However, the ECG signal must be reconstructed from the compressed one before performing diagnoses of cardiovascular diseases. The decompression step creates a slight processing delay in the diagnosis process. Thus, for remote health care monitoring and diagnosing, the compression of ECG signals is essential [10]. However, distortion due to signal compression, leads to wrong diagnosis using health care systems. In this paper, the main diagnostic parameters of ECG signal are described. It presents various heart diseases and their respective impact on ECG signals for the detection of heart abnormalities. The effect of compressing these signals before transmission on the performance of diagnosing cardiovascular abnormalities and disorders is also investigated. 


\section{ECG signal as a diagnostic tool}

A typical ECG signal for lead II has a P-wave, PR-interval, QRS-complex, ST-segment, T-wave and U-wave as illustrated in Figure (1) [11-12]. All of which are important components for diagnosing heart diseases. However, the $\mathrm{U}$ wave is invisible most of the time. The locations of the first and last samples of the P-, Q-, R-, S-, and T-waves are important features for the diagnosis of heart diseases. These feature points have a larger signal variation rate than other regions.The normal values of the ECG signal amplitudes, durations, intervals and segments are:

- The amplitude of the P-wave is less than $0.25 \mathrm{mV}(0.1-0.2 \mathrm{mV})$ and its width is less than $0.1 \mathrm{Sec} .(60-80 \mathrm{~m} \mathrm{Sec}$.).

- The amplitude of the Q-wave is less than $25 \%$ of the amplitude of the R-waves and its width is less than $0.04 \mathrm{Sec}$.

- The amplitude of the R-wave is about $1.6 \mathrm{mV}$ (it is at least $5 \mathrm{~mm}$ in amplitude in limb lead and is $10 \mathrm{~mm}$ in amplitude in pericardial lead).

- The amplitude of the $\mathrm{S}$-wave is greater than the amplitude of the $\mathrm{R}$ wave and the amplitude of the T-wave ranges from 0.1 to $0.3 \mathrm{mV}$ (its amplitude does not exceed 5 $\mathrm{mm}$ in limb lead and $10 \mathrm{~mm}$ in pericardial lead).

- The durations of the R-R and P-R intervals range from 0.4 to $1.2 \mathrm{Sec}$ and from 0.12 to $0.20 \mathrm{Sec}$ respectively.

- The durations of the QRS and Q-T intervals range from 0.08 to $0.12 \mathrm{Sec}$ and from 0.1 to 0.12 Sec respectively.

- The QRS amplitude is about $1 \mathrm{mV}$.

- The duration of the ST-segment ranges from 0.1 to $0.12 \mathrm{Sec}$.

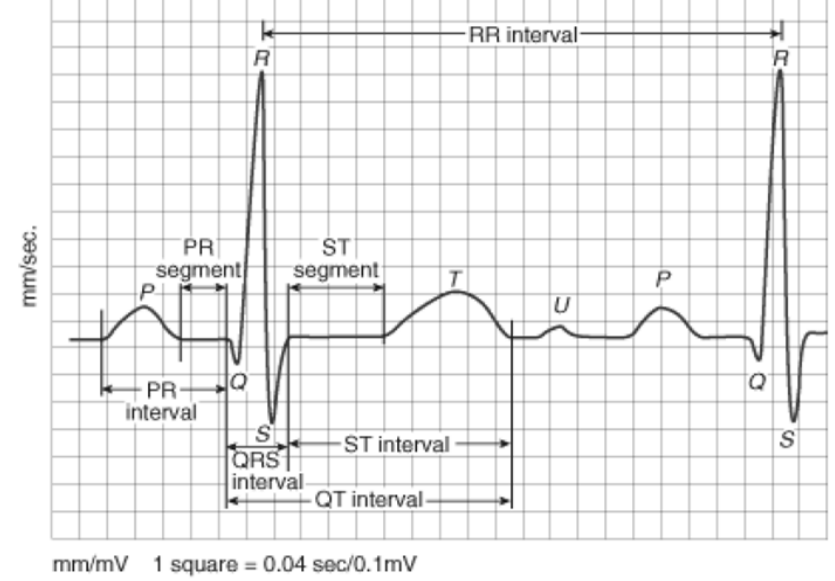

Fig. 1. Representation of normal ECG signal for lead II [12].

Characteristics of ECG waves are used for detecting the arrhythmias, conduction deficits and size or position of the chambers of the heart. ECG signal is a very weak signal and its frequency range is $0.05-100 \mathrm{~Hz}$. Moreover, most of the useful information present in the range of $0.5-45 \mathrm{~Hz}$ [13]. Under healthy conditions, the human heart chambers beat in an organized order. The importance of the ECG signal features as a diagnostic tool arises from the following two facts:

1) It can be used to determine the rate of the heart beat. 
Mohammed M. Abo-Zahhad et al., A Dedicated Diagnostic Distortion Measure for the .........

2) Various cardiac disorders including heart failure of a person can be detected.

\section{Feature extraction of ECG signal critical points}

The main effort in the feature extraction is to find the exact locations and amplitudes of all positive and negative peaks of ECG waves and complexes. The start and end points of $\mathrm{P}, \mathrm{Q}, \mathrm{R}, \mathrm{S}$, and $\mathrm{T}$ waves are important feature points for the diagnosis of heart diseases. The strategy for finding the start and end locations of these waves is to first recognize the locations and amplitudes of the peaks of the waves. First, locations and amplitudes of Rpeaks are determined. Then, the locations and amplitudes of the peaks of other waves are determined. The baseline and the ST features are relatively easily estimated later. These feature points have a larger signal variation rate than other regions. Figure (2) depicts the process of finding the feature values. It can be summarized in the following:

1. Preprocessing

2. Detection of the positions and amplitudes of the $\mathrm{R}$ - peaks $\left(\mathrm{R}_{\mathrm{pi}}, \mathrm{R}_{\mathrm{ai}}\right)$.

3. Detection of the positions and amplitudes of the Q-peaks $\left(\mathrm{Q}_{\mathrm{pi}}, \mathrm{Q}_{\mathrm{ai}}\right)$ and $\mathrm{S}$-peaks $\left(\mathrm{S}_{\mathrm{pi}}, \mathrm{S}_{\mathrm{ai}}\right)$.

4. Detection of the positions of the T-peaks $\left(T_{p i}, T_{a i}\right), U$-peaks $\left(U_{p i}, U_{a i}\right)$ and P-peaks $\left(\mathrm{P}_{\mathrm{pi}}, \mathrm{P}_{\mathrm{ai}}\right)$.

5. Detecting the start and end positions of the P-wave $\left(\mathrm{S}_{\mathrm{pi}}, \mathrm{E}_{\mathrm{pi}}\right)$, the Q-wave $\left(\mathrm{S}_{\mathrm{qi}}, \mathrm{E}_{\mathrm{q}}\right)$, the R-wave $\left(S_{\text {ri }}, E_{\mathrm{ri}}\right)$, the $S$-wave $\left(S_{\mathrm{si}}, \mathrm{E}_{\mathrm{si}}\right)$, the T-wave $\left(\mathrm{S}_{\mathrm{ti}}, \mathrm{E}_{\mathrm{ti}}\right)$, and the U-wave $\left(\mathrm{S}_{\mathrm{ui}}, \mathrm{E}_{\mathrm{ui}}\right)$.

\subsection{Preprocessing of ECG signal}

Preprocessing aims to reduce efficiently the raw data while maintaining the crucial information for further processing. The real-time recorded ECG signal is often contaminated by artifacts. Unfortunately, their frequency contents can be within the frequency band of the ECG signal. "This makes the extraction of useful information from the signal difficult" [14]. The corruption of ECG signal is due to noise and artifacts. Namely, power line interference that can be removed by notch filter, baseline drift that that can be removed by a high pass filter with $0.5 \mathrm{~Hz}$ cutoff frequency, motion artifacts that may be removed by adaptive filters and Electromyogram (EMG) interference that can be removed by morphological filter of a unit square-wave structure.

\subsection{Locating the ECG signal peaks}

The detection of the R-wave is the most important process of dividing the signal into waves. For this purpose, the R-R interval of each beat is considered to be in the range $0.4 \mathrm{Sec}$ to $1.21 \mathrm{Sec}$ samples. To find the R-peaks, a window of length greater than the maximum number of samples is adopted. In this study a window of width 500 samples is selected. The window slides to the right by one sample each time and the maximum value within the new window is calculated. To find the upper threshold levels of each beats, the sliding process is repeated until the left edge of the window. Consequently, the lower threshold levels are calculated to be half the upper threshold levels. From the obtained upper and lower threshold levels, the R peaks are determined as the maximum values of the ECG signal.

The Q- and S-peaks locations are obtained by searching for the minimum points surrounding the $\mathrm{R}$ peaks. The maximum duration of the QRS complex is $0.11 \mathrm{Sec}$ corresponding 40 samples. Similar search process to that described for detecting the Rpeaks are made in the 40 samples before and the 40 samples after the location of the Rpeak has been carried out for detecting the Q- and S-peaks. 
The T-, U- and P-peaks are located between the Q- peaks and the corresponding next S-peaks. Firstly, we define the SQ segment as the region from the location of the current Speak up to the location of the following Q-peak. The maximum and minimum points in the SQ segment are determined by adopting the same method used to find the R-peaks where the sliding window width used is selected to be equal to one-tenth the width of the SQ segment. The maximum and the minimum levels of each window are determined. The maximum points within the windows are picked and considered as the T-, U- and P-peaks after avoiding multipoint problems.

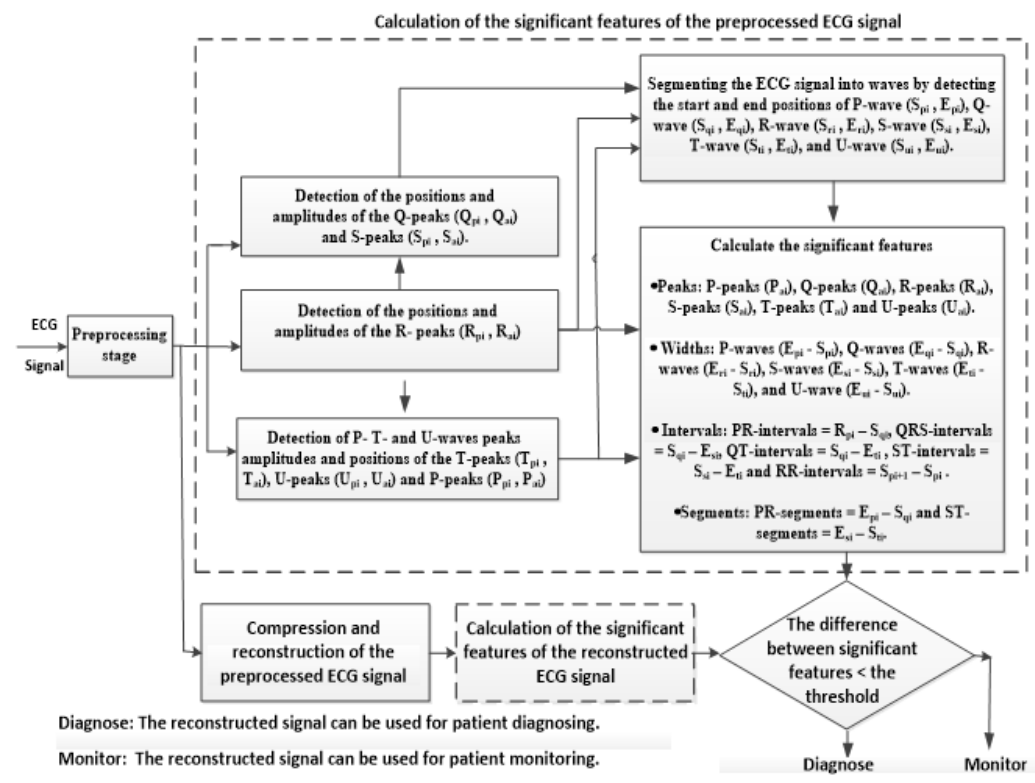

Fig. 2. The process of extracting the ECG feature values.

\subsection{Division of ECG signal into waves}

The ECG segment is defined as the period between the end of a wave and the start of the next wave. After finding the P-, Q-, R-, S-, T- and U-peaks locations, the segments PQ, ST, TU, and UP are defined as the portions of the ECG signal between the two adjacent peak locations. In each of these segments, an isoelectric wave exists. Thus, the start and end locations of other waves are determined if the start and end locations of each isoelectric wave are determined. The process of finding the start and end locations of isoelectric waves is centered on finding the longest period with the lowest standard deviation in each segment.

\section{Performance error measures}

One of the most difficult problems in ECG compression applications and reconstruction is the defining of the error criterion. The purpose of ECG compression algorithms is to remove redundancy, the irrelevant information which does not contain diagnostic information. Consequently the error criterion has to be defined such that it will measure the ability of the reconstructed signal to preserve the relevant information. The accepted way to examine the quality of the reconstructed signal is to get cardiologists' evaluations. However, in order to use such a criterion for coders design, one has to give it a mathematical diagnostic distortion measure [15]. 


\subsection{Classical error measures}

Recently, the Percent Root-mean-square Difference (PRD) measure is employed in many ECG compression algorithms [8], [10]. It is given by:

$$
P R D=\sqrt{\frac{\sum_{n=1}^{N}(x(n)-\tilde{x}(n))^{2}}{\sum_{n=1}^{N} x^{2}(n)}} \times 100
$$

where $x(n)$ is the original signal, $\tilde{x}(n)$ is the reconstructed signal, and $N$ is the length of the window over which the PRD is calculated. In literature another version of the PRD that is independently in the DC level of the original signal is defined; where $x(n)$ in the denominator of (1) is replaced by $(x(n)-\bar{x})$ and $\bar{x}$ is the average value of $x(n)$ s. The third distortion measure for comparing the original and reconstructed ECG signals is the Root Mean Square (RMS) error. It has the same definition as PRD with $x(n)$ in the denominator is replaced by N. All these error measures have many disadvantages, which all result in poor diagnostic relevance. For example, baseline drift in the reconstructed signal causes a nonzero value in all these error measures, but this distortion has no diagnostic meaning. Furthermore, every segment in the ECG signal has a different diagnostic meaning and significance. A given distortion in one segment does not necessarily have the same weight as the same distortion in another segment. For example, in many patients' ECG, the ST segment is much more diagnostically significant than the TP segment [15].

\subsection{Dedicated diagnostic distortion measures}

To overcome the limitations of the classical error performance measures, here an algorithm based on diagnostic feature extraction has been described. It investigates the effect of compressing the signals before transmission on the performance of monitoring and diagnosing CVDs. This has been carried out by introducing a suitable diagnostic distortion measure, called Dedicated Diagnostic Distortion Measure (3DM), for evaluating the performance of different ECG compression algorithms for telemedicine applications. This error measure is based on comparing the complex features of the original and reconstructed ECG signals. It takes into consideration the minimum bit rate while keeping a pre-determined diagnostic error and good reconstructed signal quality. The allowable pre-determined diagnostic error is a disease dependent and it is defined by the user of the compressor. The Diagnostic features include: duration features, amplitude features, and shape features.

The 3DM should measure the relative preservation of the relevant diagnostic information in the reconstructed signal. The diagnostic information in the ECG signals exists in the form of locations, durations, amplitudes, and shapes of the waves and complexes that exist in every ECG beat. These were chosen with the help of an experienced cardiologist. From these locations, durations, amplitudes, and shapes, the following significant features of one ECG beat are defined as follows:

Peaks $(\alpha)$ : P-peaks $\left(\mathrm{P}_{\mathrm{ai}}\right)$, Q-peaks $\left(\mathrm{Q}_{\mathrm{ai}}\right), \mathrm{R}$-peaks $\left(\mathrm{R}_{\mathrm{ai}}\right), \mathrm{S}$-peaks $\left(\mathrm{S}_{\mathrm{ai}}\right), \mathrm{T}$-peaks $\left(\mathrm{T}_{\mathrm{ai}}\right)$ and $\mathrm{U}$ peaks $\left(\mathrm{U}_{\mathrm{ai}}\right)$;

$$
\alpha=\left[\begin{array}{llllll}
\alpha_{1} & \alpha_{2} & \alpha_{3} & \alpha_{4} & \alpha_{5} & \alpha_{6}
\end{array}\right]=\left[\mathrm{P}_{\mathrm{ai}}, \mathrm{Q}_{\mathrm{ai}}, \mathrm{R}_{\mathrm{ai}}, \mathrm{S}_{\mathrm{ai}}, \mathrm{T}_{\mathrm{ai}}, \mathrm{U}_{\mathrm{ai}}\right]
$$


JES, Assiut University, Faculty of Engineering, Vol. 44, No. 2, March 2016, pp. 158 - 174

Widths: P-waves $\left(\mathrm{WP}_{\mathrm{i}}=\mathrm{E}_{\mathrm{Pi}}-\mathrm{S}_{\mathrm{Pi}}\right)$, T-waves $\left(\mathrm{WT}_{\mathrm{i}}=\mathrm{E}_{\mathrm{Ti}}-\mathrm{S}_{\mathrm{Ti}}\right)$, and U-wave $\left(\mathrm{WU}_{\mathrm{i}}=\mathrm{E}_{\mathrm{Ui}}-\mathrm{S}_{\mathrm{Ui}}\right)$;

$\beta=\left[\begin{array}{lll}\beta_{1} & \beta_{2} & \beta_{3}\end{array}\right]=\left[W P_{i}, W T_{i}, W U_{i}\right]$

Intervals: $\mathrm{PR}_{\text {intervals }}=\mathrm{R}_{\mathrm{pi}}-\mathrm{S}_{\mathrm{qi}}, \quad \mathrm{QRS}_{\text {intervals }}=\mathrm{S}_{\mathrm{qi}}-\mathrm{E}_{\mathrm{si}}, \mathrm{QT}_{\text {intervals }}=\mathrm{S}_{\mathrm{qi}}-\mathrm{E}_{\mathrm{ti}}, \mathrm{ST}_{\text {intervals }}=\mathrm{S}_{\mathrm{si}}-\mathrm{E}_{\mathrm{ti}}$ and $\mathrm{RR}_{\text {intervals }}=\mathrm{S}_{\mathrm{pi}+1}-\mathrm{S}_{\mathrm{pi}}$;

$\gamma=\left[\begin{array}{lllll}\gamma_{1} & \gamma_{2} & \gamma_{3} & \gamma_{4} & \gamma_{5}\end{array}\right]=\left[\mathrm{PR}_{\text {intervals }}, \mathrm{QRS}_{\text {intervals }}, \mathrm{QT}_{\text {intervals, }}, \mathrm{ST}_{\text {intervals }}, \mathrm{RR}_{\text {intervals }}\right]$

Segments: $\mathrm{PR}_{\text {segments }}=\mathrm{E}_{\mathrm{pi}}-\mathrm{S}_{\mathrm{qi}}$ and $\mathrm{ST}_{\text {segments }}=\mathrm{E}_{\mathrm{si}}-\mathrm{S}_{\mathrm{ti}}$;

$\mu=\left[\begin{array}{ll}\mu_{1} & \mu_{2}\end{array}\right]=\left[\mathrm{PR}_{\text {segments }}, \mathrm{ST}_{\text {segments }}\right]$

From equations (3)-(6), the features vector $f$ is formed as

$$
f=\left[\begin{array}{llll}
\alpha & \beta & \gamma & \mu
\end{array}\right]
$$

Consultation with cardiologists reveals that of the 12 CVDs considered in this paper, the peak of the U-wave $\left(\alpha_{6}=\mathrm{U}\right.$-peaks $\left.\left(\mathrm{U}_{\mathrm{ai}}\right)\right)$, the widths of both $\mathrm{T}$ - and U-waves $\left(\beta_{2}=\mathrm{T}\right.$-waves $\left(\mathrm{WT}_{\mathrm{i}}=\mathrm{E}_{\mathrm{Ti}}-\mathrm{S}_{\mathrm{Ti}}\right)$, and $\beta_{3}=\mathrm{U}$-wave $\left(\mathrm{WU}_{\mathrm{i}}=\mathrm{E}_{\mathrm{Ui}}-\mathrm{S}_{\mathrm{Ui}}\right)$;), the intervals $\gamma_{3}=\mathrm{QT}_{\text {intervals }}$ and $\gamma_{4}=\mathrm{ST}_{\text {intervals }}$ as well as the PR-segment $\mu_{1}=\mathrm{PR}_{\text {segments }}$ have no help in the diagnoses of such diseases. Thus, the features vector $f$ is expressed as

$$
f=\left[\begin{array}{llllllllll}
\alpha_{1} & \alpha_{2} & \alpha_{3} & \alpha_{4} & \alpha_{5} & \beta_{1} & \gamma_{1} & \gamma_{2} & \gamma_{5} & \mu_{2}
\end{array}\right]
$$

In addition, not all the remaining features listed in equation (8) have diagnostic information for all diseases. Table (2) illustrates the features that should be used for diagnosing the most important 12 CVDs.

The empty entries in the table mean that the corresponding features are not indicative of a disease. For example, the features $\alpha_{1}, \beta_{1}, \gamma_{2}$ and $\gamma_{5}$ are used for the diagnoses of Sinus Bradycardia and the remaining features $\left(\alpha_{2}, \alpha_{3}, \alpha_{4}, \alpha_{5}, \gamma_{1}\right.$ and $\left.\mu_{2}\right)$ are of no use for that disease.

Table 2.

Features of different CVDs.

\begin{tabular}{|c|c|c|c|c|c|c|c|c|c|c|}
\hline $\begin{array}{c}\text { Features } \\
\text { Diseases }\end{array}$ & $\alpha_{1}$ & $\alpha_{2}$ & $\alpha_{3}$ & $\alpha_{4}$ & $\alpha_{5}$ & $\beta_{1}$ & $\gamma_{1}$ & $\gamma_{2}$ & $\gamma_{5}$ & $\mu_{2}$ \\
\hline $\begin{array}{c}\text { Sinus } \\
\text { Bradycardia }\end{array}$ & $\sqrt{ }$ & & & & & $\sqrt{ }$ & & $\sqrt{ }$ & $\sqrt{ }$ & \\
\hline $\begin{array}{c}\text { Sinus } \\
\text { Tachycardia }\end{array}$ & $\sqrt{ }$ & & & & $\sqrt{ }$ & & $\sqrt{ }$ & $\sqrt{ }$ & \\
\hline $\begin{array}{c}\text { Sinus } \\
\text { Arrhythmia }\end{array}$ & $\sqrt{ }$ & & & & $\sqrt{ }$ & & $\sqrt{ }$ & $\sqrt{ }$ & \\
\hline $\begin{array}{c}\text { Atrial } \\
\text { Premature Beat } \\
\text { (APB) }\end{array}$ & $\sqrt{ }$ & & & & $\sqrt{ }$ & & $\sqrt{ }$ & & \\
\hline Atrial Fibrillation & $\sqrt{ }$ & & & & $\sqrt{ }$ & & & & $\sqrt{ }$ & \\
\hline Atrial Flutter & $\sqrt{ }$ & & & & $\sqrt{ }$ & & & & & \\
\hline $\begin{array}{c}\text { Right Bundle } \\
\text { Branch Block } \\
\text { (RBBB) }\end{array}$ & & $\sqrt{ }$ & $\sqrt{ }$ & $\sqrt{ }$ & $\sqrt{ }$ & & $\sqrt{ }$ & & & \\
\hline
\end{tabular}


Mohammed M. Abo-Zahhad et al., A Dedicated Diagnostic Distortion Measure for the .........

\begin{tabular}{|c|c|c|c|c|c|c|c|c|c|c|}
\hline $\begin{array}{c}\text { Features } \\
\text { Diseases }\end{array}$ & $\alpha_{1}$ & $\alpha_{2}$ & $\alpha_{3}$ & $\alpha_{4}$ & $\alpha_{5}$ & $\beta_{1}$ & $\gamma_{1}$ & $\gamma_{2}$ & $\gamma_{5}$ & $\mu_{2}$ \\
\hline $\begin{array}{c}\text { Left Bundle } \\
\text { Branch Block } \\
\text { (LBBB) }\end{array}$ & & $\sqrt{ }$ & $\sqrt{ }$ & $\sqrt{ }$ & $\sqrt{ }$ & & $\sqrt{ }$ & & & $\sqrt{ }$ \\
\hline $\begin{array}{c}\text { Ventricular } \\
\text { Tachycardia }\end{array}$ & & $\sqrt{ }$ & $\sqrt{ }$ & $\sqrt{ }$ & & & $\sqrt{ }$ & $\sqrt{ }$ & $\sqrt{ }$ & \\
\hline $\begin{array}{c}\text { Different } \\
\text { Degrees of Heart } \\
\text { Block }\end{array}$ & $\sqrt{ }$ & & & & & $\sqrt{ }$ & $\sqrt{ }$ & $\sqrt{ }$ & $\sqrt{ }$ & \\
\hline $\begin{array}{c}\text { ST-elevation } \\
\text { myocardial } \\
\text { infarction }\end{array}$ & & & & & $\sqrt{ }$ & & & & & $\sqrt{+}$ \\
\hline $\begin{array}{c}\text { Non ST- } \\
\text { elevation } \\
\text { myocardial } \\
\text { infarction }\end{array}$ & & & & & & & & & $\sqrt{ }-$ \\
\hline
\end{tabular}

To consider certain features for certain disease, the vector $f$, described by equation (8), is multiplied by the a weighting vector $W$ described by:

$$
W=\left[\begin{array}{llllllllll}
w_{1} & w_{2} & w_{3} & w_{4} & w_{5} & w_{6} & w_{7} & w_{8} & w_{9} & w_{10}
\end{array}\right]
$$

This results the weighted feature vector $f_{w}$ that is described by

$$
f_{w}=f * W^{T}
$$

Where, ${ }^{*}$ denotes element-by-element multiplication. If the $\mathrm{i}^{\text {th }}$ feature is not important for certain disease, $w_{i}$ is set to zero. For example, for Sinus Bradycardia disease $W$ is given by

$$
W=\left[\begin{array}{llllllllll}
w_{1} & 0 & 0 & 0 & 0 & w_{6} & 0 & w_{8} & w_{9} & 0
\end{array}\right]
$$

For other diseases, similar constructions of $\mathrm{W}$ to that given by equation (11) can be used. The above analysis is carried out for a signal having only one beat. In this case, the dedicated diagnostic distortion measure, 3DM, is given by:

$$
3 \mathrm{DM} \%=\sqrt{\frac{\sum_{j=1}^{10}\left(f_{w}(j)-\tilde{f}_{w}(j)\right)^{2}}{\sum_{j=1}^{10} f_{w}{ }^{2}(j)}} \times 100 \%
$$

Where, $f_{w}(j)$ and $\tilde{f}_{w}(j)$ are the $\mathrm{j}^{\text {th }}$ weighted feature value of the original and reconstructed signals respectively. For lengthy signal contains $N_{\text {beats }}$ beats, the 3DM is given by: 


$$
3 \mathrm{DM} \%=\sqrt{\frac{\sum_{\mathrm{i}=1}^{\text {Nbeats }} \sum_{j=1}^{10}(f(i, j)-\tilde{f}(i, j))^{2}}{\sum_{\mathrm{i}=1}^{\text {Nbeats }} \sum_{j=1}^{10}(f(i, j))^{2}}} \times 100 \%
$$

Where, $f(i, j)$ and $\tilde{f}(i, j)$ are respectively the $\mathrm{j}^{\text {th }}$ weighted feature value of the original and reconstructed signals for the $\mathrm{i}^{\text {th }}$ beat.

\section{3. Mean opinion score distortion measure}

To test the quality of the reconstructed ECG signals, it is necessary to use real clinical evaluation carried out by cardiologists in order to validate compression methods. For example, the MOS test was presented in [15] as a real clinical evaluation test. In this test both the original and reconstructed ECG signals of a certain record are printed in a paper or displayed in electronic form. Then cardiologists are asked to evaluate these signals. For every tested signal, the evaluators are asked to answer some questions about the quality of the reconstructed ECG signal. These questions are listed in Table (3).

\section{Table 3.}

MOS test questionnaire.

Evaluation of original and reconstructed ECG signals extracted from MIT-BIH record .......

1- Cardiologist Name: Cardiologist ID

2- Degree of similarity between the original and reconstructed signals (select any one number)

\begin{tabular}{|c|c|c|c|c|}
\hline 1 & 2 & 3 & 4 & 5 \\
\hline $\begin{array}{c}\text { Completely } \\
\text { different }\end{array}$ & $\ldots \ldots$ & $\ldots \ldots$ & $\ldots \ldots$ & $\begin{array}{c}\text { Completely } \\
\text { identical }\end{array}$ \\
\hline
\end{tabular}

3- Would you give a different diagnosis with the tested signal if you hadn't seen the original signal? (circle Yes or No) [15].

\begin{tabular}{|l|c|}
\hline \multicolumn{1}{|c|}{ Yes } & No \\
\hline
\end{tabular}

The traditional definition of the percentage MOS error for any tester $\mathrm{k}$ is given by:

$$
\operatorname{MOS}(k)=\left(\text { factor } x \frac{5-C}{5}+(1-\text { factor }) x(1-D)\right) \times 100
$$

where, $C$ is a five scale that measures the similarity between original and reconstructed signals ( 1 for completely different signals and 5 for completely identical signals).

$D$ is the answer to the Boolean question about the diagnosis (0-YES, 1-NO).

factor is a weighting coefficient between the measure of similarity and the Boolean question (0 to less than 1$)$.

In this paper, the cardiologists are asked to evaluate the individual features rather than evaluating the complete ECG signal at once. In this case, the second and third items of the 
questionnaire listed in Table (3) are asked $N f$ times; where $N f$ is the number of considered features. Thus the definition of the percentage MOS error for any tester $k$ described by equation (14) is modified to

$$
\operatorname{MOS}(k)=\sum_{i}^{N f}\left(\text { factor } x \frac{5-C(i)}{5}+(1-\text { factor }) x(1-D(i))\right) \times 100
$$

In both cases, the overall mean percentage MOS error is determined from the following equation.

$$
\operatorname{MOS} \%=\frac{1}{N_{V}} \sum_{k=1}^{N_{V}} \operatorname{MOS}(k) \%
$$

where, $N_{V}$ is the number of evaluators.

\section{Compression of ECG signal based on CS and the extraction of significant features}

In technical literature, Compressive Sensing (CS) has been introduced to overcome the limitations of the classical sampling theory pioneered by Nyquist and Shannon [16]. The classical sampling theory relies on the assumption that the signals to be acquired are bandlimited to a maximum frequency: the Nyquist frequency. Even if this hypothesis does not hold, the signals can simply be low-pass filtered before being sampled at a rate at least twice the Nyquist frequency. CS is a revolutionary signal acquisition scheme that allows a signal to be acquired and accurately reconstructed with significantly fewer samples than required by Nyquist-rate sampling. Unlike Nyquist sampling, which relies on the maximum rate-of-change of a signal, CS relies on the maximum rate-of-information in a wide range of applications; especially in telediagnosing of CVDs [17]-[18]. In [19], a brief overview of the basic principles of CS is provided to form the basis of most signal processing applications.

In [20] compression of ECG signal based on CS and the extraction of significant features has been introduced. It is adopted here for testing the performance evaluation of the 3DM. It is based on improving the ECG signal sparsity using QRS-complex estimation based on the peaks and locations of Q, R and S waves. Then, the estimated QRS-complex is subtracted from the original ECG signal and the resulting differential signal is manipulated using CS technique as shown in Figure (3) where fewer measurements are determined from the resulting error signal [21-22]

\section{Illustrative examples}

In this paper, records extracted from the MIT-BIH arrhythmia database [23] has been adopted for the performance evaluation of the adopted ECG compression technique described in section 5. This database comprises 48 ECG recordings of 30 minutes duration selected from 24 hours recordings. Each ECG signal is digitized at 360 samples per second per channel with 11-bit resolution over a $10 \mathrm{mV}$ range. The subjects were 25 men aged 32 to 89 years, and 22 women aged 23 to 89 years. This database has different morphologies of Premature Ventricular Contractions (PVCs) and Normal Beats (NBs). In most records of the database, channel one is a modified limb lead II, and channel two is usually a modified lead V1 (occasionally V2 or V5). This database contains different types of arrhythmias.

Example 1: To illustrate the calculation of the feature values and the dedicated diagnostic distortion measure 3DM, consider the 1460 sample signal shown in Figure (4) extracted from record 103 of MIT-BIH database. The signal is compressed with $\mathrm{CR}=11.89$ using the method described in [24] with $\mathrm{PRD}=7.08 \%$. The reconstructed signal is shown in Figure (5). Tables (4) 
JES, Assiut University, Faculty of Engineering, Vol. 44, No. 2, March 2016, pp. 158 - 174

and (5) include the amplitudes and the locations of the signal waves for the original and the reconstructed ECG signals respectively. Tables (6) and (7) include the start and end locations of the P-waves, QRS-complexes, T-waves and U-waves for the original and the reconstructed ECG signals respectively. Table (8) includes the 10 features for the original and reconstructed signals. Finally, the 3DM\% of different beat and the complete signal are given in Table (9).

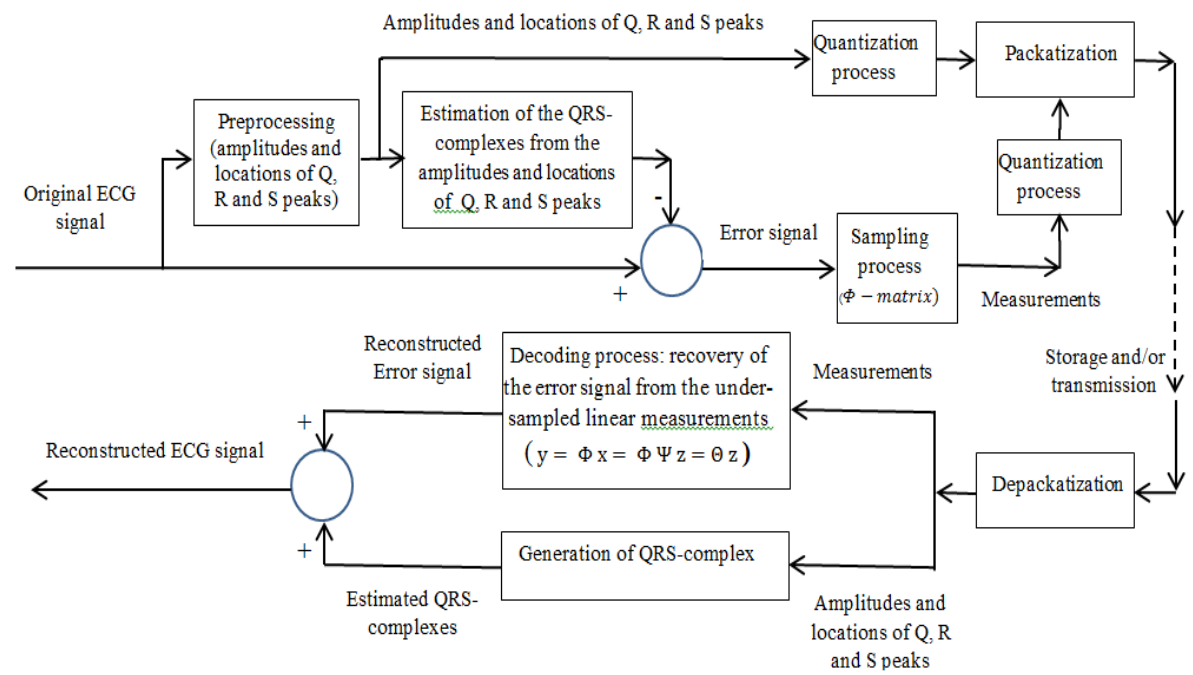

Fig. 3. Block diagram of the adopted CS based compression technique [22].

Table 4.

Amplitudes and locations of the waves peaks in the original signal.

\begin{tabular}{|c|c|c|c|c|c|c|c|c|c|c|c|c|}
\hline \multirow{2}{*}{$\begin{array}{c}\text { Beat } \\
\text { number }\end{array}$} & \multicolumn{2}{|c|}{ P-wave } & \multicolumn{2}{c|}{ Q-wave } & \multicolumn{2}{c|}{ R-wave } & \multicolumn{2}{c|}{ S-wave } & \multicolumn{2}{c|}{ T-wave } & \multicolumn{2}{c|}{ U-wave } \\
\cline { 2 - 15 } & Loc. & Amp. & Loc. & Amp. & Loc. & Amp. & Loc. & Amp. & Loc. & Amp. & Loc. & Amp. \\
\hline 1 & 206 & -0.103 & 255 & -0.295 & 266 & 0.925 & 275 & -0.263 & 362 & 0.058 & 430 & -0.148 \\
\hline 2 & 517 & -0.125 & 566 & -0.318 & 577 & 0.920 & 584 & -0.275 & 667 & 0.038 & 729 & -0.155 \\
\hline 3 & 817 & -0.14 & 866 & -0.300 & 878 & 0.850 & 885 & -0.288 & 969 & 0.030 & 1050 & -0.138 \\
\hline 4 & 1123 & -0.118 & 1171 & -0.293 & 1182 & 0.910 & 1189 & -0.280 & 1274 & 0.068 & 1346 & -0.135 \\
\hline
\end{tabular}

Table 5.

Amplitudes and locations of the waves peaks in the reconstructed signal.

\begin{tabular}{|c|c|c|c|c|c|c|c|c|c|c|c|c|}
\hline \multirow{2}{*}{$\begin{array}{c}\text { Beat } \\
\text { number }\end{array}$} & \multicolumn{2}{|c|}{ P-wave } & \multicolumn{2}{c|}{ Q-wave } & \multicolumn{2}{c|}{ R-wave } & \multicolumn{2}{c|}{ S-wave } & \multicolumn{2}{c|}{ T-wave } & \multicolumn{2}{c|}{ U-wave } \\
\cline { 2 - 37 } & Loc. & Amp. & Loc. & Amp. & Loc. & Amp. & Loc. & Amp. & Loc. & Amp. & Loc. & Amp. \\
\hline 1 & 207 & -0.111 & 255 & -0.298 & 266 & 0.923 & 274 & -0.268 & 362 & 0.006 & 423 & -0.136 \\
\hline 2 & 518 & -0.134 & 566 & -0.328 & 577 & 0.923 & 584 & -0.278 & 668 & 0.031 & 719 & -0.138 \\
\hline 3 & 837 & -0.162 & 866 & -0.308 & 878 & 0.823 & 885 & -0.318 & 967 & 0.030 & 1068 & -0.149 \\
\hline 4 & 1129 & -0.145 & 1171 & -0.338 & 1182 & 0.873 & 1189 & -0.308 & 1274 & 0.064 & 1369 & -0.138 \\
\hline
\end{tabular}

\section{Table 6.}

Start and end locations of the P-waves, QRS-complexes, T-waves and U-waves for the original.

\begin{tabular}{|c|c|c|c|c|c|c|c|c|}
\hline \multirow{2}{*}{$\begin{array}{c}\text { Beat } \\
\text { number }\end{array}$} & \multicolumn{2}{|c|}{ P-wave } & \multicolumn{2}{c|}{ QRS-complex } & \multicolumn{2}{c|}{ T-wave } & \multicolumn{2}{c|}{ U-wave } \\
\cline { 2 - 9 } & Start & End & Start & End & Start & End & Start & End \\
\hline 1 & 192 & 225 & 252 & 285 & 331 & 387 & 423 & 455 \\
\hline 2 & 501 & 536 & 563 & 591 & 635 & 689 & 722 & 757 \\
\hline 3 & 803 & 837 & 864 & 892 & 936 & 992 & 1035 & 1069 \\
\hline 4 & 1108 & 1142 & 1168 & 1195 & 1240 & 1299 & 1337 & 1369 \\
\hline
\end{tabular}


Table 7.

Start and end locations of the P-waves, QRS-complexes, T-waves and U-waves for the reconstructed 1460 samples.

\begin{tabular}{|c|c|c|c|c|c|c|c|c|}
\hline \multirow{2}{*}{$\begin{array}{c}\text { Beat } \\
\text { number }\end{array}$} & \multicolumn{2}{|c|}{ P-wave } & \multicolumn{2}{|c|}{ QRS-complex } & \multicolumn{2}{c|}{ T-wave } & \multicolumn{2}{c|}{ U-wave } \\
\cline { 2 - 8 } & Start & End & Start & End & Start & End & Start & End \\
\hline 1 & 169 & 225 & 251 & 285 & 331 & 390 & 423 & 455 \\
\hline 2 & 505 & 537 & 563 & 591 & 635 & 692 & 720 & 720 \\
\hline 3 & 779 & 837 & 854 & 887 & 930 & 1016 & 1069 & 1069 \\
\hline 4 & 1102 & 1142 & 1165 & 1194 & 1239 & 1335 & 1370 & 1371 \\
\hline
\end{tabular}

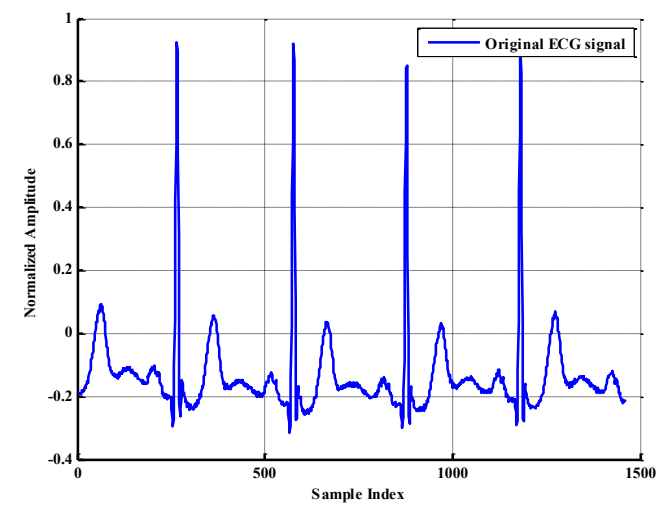

Fig. 4. Original ECG signal used for the illustrating the 3DM\% calculations [23].

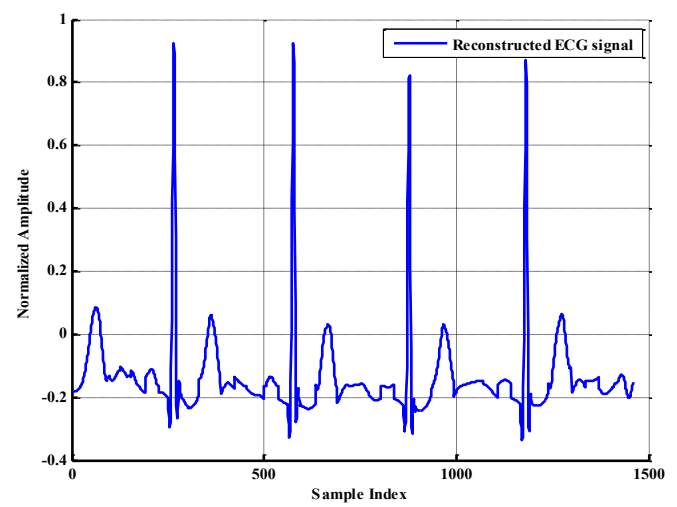

Fig. 5. Reconstructed ECG signal used for the illustrating the 3DM\% calculations [24].

Table 8.

The 10 features for the original and reconstructed ECG signals.

\begin{tabular}{|c|c|c|c|c|c|c|c|c|c|c|c|}
\hline Signals & $\begin{array}{c}\text { Beat } \\
\text { number }\end{array}$ & $\alpha_{1}$ & $\alpha_{2}$ & $\alpha_{3}$ & $\alpha_{4}$ & $\alpha_{5}$ & $\beta_{1}$ & $\gamma_{1}$ & $\gamma_{2}$ & $\gamma_{5}$ & $\mu_{2}$ \\
\hline \multirow{4}{*}{ Original } & 1 & -0.103 & -0.295 & 0.925 & -0.263 & 0.058 & 33 & 60 & 60 & 311 & 44 \\
\cline { 2 - 12 } & 2 & -0.125 & -0.318 & 0.920 & -0.275 & 0.038 & 35 & 61 & 18 & 301 & 44 \\
\cline { 2 - 12 } & 3 & -0.14 & -0.300 & 0.850 & -0.288 & 0.030 & 34 & 59 & 19 & 304 & 44 \\
\cline { 2 - 12 } & 4 & -0.118 & -0.293 & 0.910 & -0.280 & 0.068 & 34 & 20 & 18 & 0 & 45 \\
\hline \multirow{5}{*}{ Reconstructed } & 1 & -0.111 & -0.298 & 0.923 & -0.268 & 0.0061 & 56 & 59 & 59 & 311 & 46 \\
\cline { 2 - 12 } & 2 & -0.134 & -0.328 & 0.923 & -0.278 & 0.031 & 32 & 41 & 18 & 301 & 44 \\
\cline { 2 - 12 } & 3 & -0.162 & -0.308 & 0.823 & -0.318 & 0.030 & 58 & 53 & 19 & 304 & 43 \\
\cline { 2 - 11 } & 4 & -0.145 & -0.338 & 0.873 & -0.308 & 0.064 & 40 & 19 & 18 & 45 \\
\hline
\end{tabular}


JES, Assiut University, Faculty of Engineering, Vol. 44, No. 2, March 2016, pp. $158-174$

Table 9.

3DM\% of different beat and the complete signal.

\begin{tabular}{|c|c|c|c|c|c|}
\hline & \multicolumn{4}{|c|}{ Individual Beats } & Complete signal \\
\hline Beat number & 1 & 2 & 3 & 4 & All beats \\
\hline 3DM\% & $4.1665 \%$ & $3.6429 \%$ & $4.4599 \%$ & $1.0958 \%$ & $7.1939 \%$ \\
\hline
\end{tabular}

Example 2: This example presents the analysis of four specific heart diseases; namely APB, LBBB, RBBB and PVC. It illustrates the calculation of 3DM\%, PRD\% and MOS\% of the feature values of the original and compressed signals for beating representing persons with these diseases as well as healthy person with Normal Beats (NBs). It is necessary to mention that the reconstructed signal should be suitable for the cardiologist for the analysis and interpretation about the health of the patients. This would be helpful for better diagnosis of diseases with low cost suitable processing tools and techniques. It provides the cardiologists to have more understanding of signal and they could serve the mankind to have a better world. For this purpose, 18 records for patients suffering from the 4 mentioned diseases and 6 records for healthy persons are investigated. Moreover, compression method based on CS and the extraction of significant features described in section 5 is adopted. For all considered records, the signal is of length 9216 samples and Debauches' discrete wavelet transform filter of the fourth order (db4) has been used to achieve high CR, low PRD and low 3DM. The decomposition process has been carried out up to the $5^{\text {th }}$ level. For the MOS test ten the cardiologists are asked to evaluate the individual features as well as the complete ECG signal. Then, equations (14)-(16) have been used for finding the MOS\%, where the scaling factor is set to half (factor $=0.5$ ).

Firstly, the proposed method has been tested for 5 MIT-BIH records listed in the second column of Table (10); namely records 209, 200, 111 and 118 for patients suffering from APB (APC), PVC, LBBB, and RBBB diseases respectively and record 100 for healthy people with NBs. These records are used for the assessment of the proposed method. Moreover, the MIT-BIH database lists the diseases of the corresponding records. The prior knowledge of the disease before transmission is not necessary; since the evaluation is decompressing the signal at the doctor side. The table includes the values of the 3DM\%, MOS\%, PRD\% and CR for each record. Secondly, the method has been tested for the 24 MIT-BIH records listed in Table (11). Moreover, the average values of the 3DM\%, MOS\%, PRD\% and CR for each group of records are included. From the obtained results, it can be noticed that the obtained values of the 3DM\% are consistent with that of the MOS\%; where the ratio between both of them is almost constant for all considered records. However, the obtained values of the PRD\% don't reflect the results obtained by the cardiologists which have the highest accuracy. This means that for all tested signals the evaluation based on the proposed 3DM is acceptable from the point view of the cardiologists' evaluators and there is no loss in the clinical information of the ECG signal.

\section{Conclusion}

The increasing burden associated with CVD necessitates the investigation of innovative methods to provide evidence-based identification of diseases. This paper presents automatic ECG signal interpretations used for detecting cardiovascular diseases. It investigates the effect of compressing ECG signals on the performance of diagnosing CVDs. This has been performed by introducing a suitable diagnostic distortion measure, 3DM, based on comparing the PQRST complex features of the original and reconstructed 
ECG signals. It takes into consideration the minimum bit rate while keeping a predetermined diagnostic error and good reconstructed signal quality. The allowable predetermined diagnostic error is a disease dependent. The diagnostic features considered in this paper include duration features, amplitude features and shape features. To test the effectiveness of the proposed method, 18 records of patients suffering from 4 CVDs and 6 records for healthy persons have been investigated. Consultations with cardiologists through MOS test show that the proposed 3DM is most informative compared to the wellknown PRD measure for evaluating compression techniques oriented for CVDs monitoring and diagnosing. The price paid for the usage of 3DM as a good performance metric is the computational complexity in extracting the diagnostic features. In many cases the physician is interested on both compression and analysis of ECG signals. Therefore, the extracted features can be used for patients diagnosing.

The adoption of RMSE or PRD as a measure of the accuracy of compressiondecompression techniques as it does not require any prior knowledge of the ECG diagnostic decision is questionable. This is due to the fact that both of them are calculated as an average all over the signal length. However, the diagnostic information is concentrated at some intervals in the signal. Thus, although the accuracy of the assessment of the suggested technique depends on the possible accurate determination of the disease features, it has been proved by cardiologists adopting the MOS test that 3DM is a good performance metric for CVDs. The problem of inaccurate estimation of the computed features and consequently the 3DM calculation due to inability of removing of power line interference and the base line wandering due to patient movement will be treated in the future.

Table 10.

Beat types and evaluation of the proposed 3DM method for selected MIT-BIH records

\begin{tabular}{|c|c|c|c|c|c|c|c|c|c|c|c|c|c|c|c|c|}
\hline \multirow[b]{2}{*}{$\begin{array}{c}\text { Diseases } \\
\text { (Beat } \\
\text { Type) }\end{array}$} & \multirow[b]{2}{*}{$\begin{array}{l}\text { Record } \\
\text { Number }\end{array}$} & \multirow[b]{2}{*}{ Signal } & \multicolumn{10}{|c|}{ Features } & \multirow[b]{2}{*}{$\begin{array}{l}3 \mathrm{D} \\
\mathrm{M} \%\end{array}$} & \multirow[b]{2}{*}{$\begin{array}{c}\text { PR } \\
\text { D } \\
\%\end{array}$} & \multirow[b]{2}{*}{$\begin{array}{l}\mathrm{MO} \\
\mathrm{S} \%\end{array}$} & \multirow[b]{2}{*}{$\mathrm{CR}$} \\
\hline & & & $\alpha_{1}$ & $\alpha_{2}$ & $\alpha_{3}$ & $\alpha_{4}$ & $\alpha_{5}$ & $\beta_{1}$ & $\gamma_{1}$ & $\gamma_{2}$ & $\gamma_{5}$ & $\mu_{2}$ & & & & \\
\hline \multirow{2}{*}{ NB } & \multirow{2}{*}{100} & Original & $\sqrt{ }$ & $\sqrt{ }$ & $\sqrt{ }$ & $\sqrt{ }$ & $\sqrt{ }$ & $\sqrt{ }$ & $\sqrt{ }$ & $\sqrt{ }$ & $\sqrt{ }$ & $\sqrt{ }$ & \multirow{2}{*}{0.87} & \multirow{2}{*}{1.12} & \multirow{2}{*}{96.4} & \multirow{2}{*}{18.3} \\
\hline & & Reconstructed & $\sqrt{ }$ & $\sqrt{ }$ & $\sqrt{ }$ & $\sqrt{ }$ & $\sqrt{ }$ & $\sqrt{ }$ & $\sqrt{ }$ & $\sqrt{ }$ & $\sqrt{ }$ & $\checkmark$ & & & & \\
\hline \multirow[b]{2}{*}{ APB } & \multirow[b]{2}{*}{209} & Original & $\sqrt{ }$ & & & & & $\sqrt{ }$ & & $\sqrt{ }$ & & & \multirow[b]{2}{*}{0.84} & \multirow[b]{2}{*}{1.17} & \multirow[b]{2}{*}{93.1} & \multirow[b]{2}{*}{20.4} \\
\hline & & Reconstructed & $\sqrt{ }$ & & & & & $\sqrt{ }$ & & $\sqrt{ }$ & & & & & & \\
\hline \multirow{2}{*}{ PVC } & \multirow{2}{*}{200} & Original & & $\sqrt{ }$ & $\sqrt{ }$ & $\sqrt{ }$ & & & $\sqrt{ }$ & $\sqrt{ }$ & $\sqrt{ }$ & & \multirow{2}{*}{0.80} & \multirow{2}{*}{2.18} & \multirow{2}{*}{89.4} & \multirow{2}{*}{17.9} \\
\hline & & Reconstructed & & $\sqrt{ }$ & $\sqrt{ }$ & $\sqrt{ }$ & & & $\sqrt{ }$ & $\sqrt{ }$ & $\sqrt{ }$ & & & & & \\
\hline \multirow{2}{*}{ LBBB } & \multirow{2}{*}{111} & Original & & $\sqrt{ }$ & $\sqrt{ }$ & $\sqrt{ }$ & $\sqrt{ }$ & & $\sqrt{ }$ & & & & \multirow{2}{*}{0.77} & \multirow{2}{*}{1.37} & \multirow{2}{*}{85.7} & \multirow{2}{*}{18.9} \\
\hline & & Reconstructed & & $\sqrt{ }$ & $\sqrt{ }$ & $\sqrt{ }$ & $\sqrt{ }$ & & $\sqrt{ }$ & & & & & & & \\
\hline \multirow[t]{2}{*}{ RBBB } & \multirow{2}{*}{118} & Original & & $\sqrt{ }$ & $\sqrt{ }$ & $\sqrt{ }$ & $\sqrt{ }$ & & $\sqrt{ }$ & & & & \multirow{2}{*}{0.83} & 247 & 912 & 195 \\
\hline & & Reconstructed & & $\sqrt{ }$ & $\sqrt{ }$ & $\sqrt{ }$ & $\sqrt{ }$ & & $\sqrt{ }$ & & & & & 2.47 & 31.2 & 18. \\
\hline
\end{tabular}

Table 11.

Evaluation of the proposed 3DM method for selected MIT-BIH records

\begin{tabular}{|c|c|c|c|c|c|}
\hline \multirow{2}{*}{$\begin{array}{c}\text { Diseases } \\
\text { (Beat Type) }\end{array}$} & \multirow{2}{*}{ MIT-BIH Records } & \multicolumn{4}{|c|}{ Average values } \\
\hline & & 3DM\% & PRD\% & MOS\% & $\mathrm{CR}$ \\
\hline NB & $100,105,106,116,233,234$ & 0.903 & 1.48 & 95.3 & 17.6:1 \\
\hline APB & 209,222 & 0.865 & 1.19 & 92.1 & $19.5: 1$ \\
\hline PVC & $\begin{array}{c}119,200,203,208,210,213, \\
221,223\end{array}$ & 0.810 & 2.38 & 82.5 & $17.2: 1$ \\
\hline LBBB & 111,207, and 214 & 0.830 & 1.94 & 87.1 & 18.1:1 \\
\hline RBBB & $118,124,212,231$, and 232 & 0.845 & 1.79 & 90.7 & 19.3:1 \\
\hline
\end{tabular}




\section{Acknowledgements}

We deeply acknowledge Faculty of Engineering, Minia University, Egypt for funding this research project. The valuable comments of the two reviewers are highly appreciated.

\section{REFERENCES}

[1] A. L. Goldberger. Clinical electrocardiography: a simplified approach. Mosby Elsevier, 2006.

[2] G. Bakul, and U. S. Tiwary, "Automated risk identification of myocardial infarction using relative frequency band coefficient (RFBC) features from ECG," The Open Biomedical Engineering Journal, 4, pp. 217-222, 2010.

[3] L. Sornmo and P. Laguna. Bioelectrical signal processing in cardiac and neurological applications. Academic Press, 2005.

[4] B. Neogi, S. Ghosal, S. Mukherjee, S. Ghosh, and A. Das, "Study of Cardiovascular Dynamics with Recursive Simulator Generation Approach," International Journal of Information Technology Technology and Knowledge Management, Vol. 4, No. 1, pp. 157-161, January-June 2011.

[5] C. Wen, M. F. Yeh, K. C. Chang, R.G. Lee "Real-time ECG telemonitoring system design with mobile phone platform" Journal of Measurement, vol. 41, pp. 463-470, May 2007.

[6] N. S. Rani, K.Vimala and Dr.V.Kalaivani, "Health care monitoring for the CVD detection using soft computing techniques," International Journal in Foundations of Computer Science and Technology (IJFCST), vol. 3, no. 4, pp. 21-30, July 2013.

[7] S. Bhattacharjee, A. Mazumder, Z. Das, L. Kumar, K. Shree, B. Neogi, "Study on cardiovascular diseases with compression of generalized ECG signal to support biomedical advancement" Inter. J. Advanced Research in Electrical, Electronics and Instrumentation Engineering, pp. 5213-5220, vol. 2, no. 10, Oct. 2013.

[8] M. Abo-Zahhad, "ECG signal compression using discrete wavelet transform," Chapter in "Discrete wavelet transforms - theory and applications," Edited by Juuso T. Olkkonen, InTech, April, 2011.

[9] F. Sufi, "Efficient and secured wireless monitoring systems for detection of cardiovascular diseases," College of Science, Engineering and Health (SEH), RMIT University, Melbourne, Victoria, Australia, March 2011.

[10] M. Abo-Zahhad, A. F. Al-Ajlouni, S. M. Ahmed and R. J. Schilling, "A new algorithm for the compression of ECG signals based on mother wavelet parameterization and best-threshold levels selection," Digital Signal Processing, vol. 23, no. 3, pp. 1002-1011, May 2013.

[11] W. C. Mueller, "Arrhythmia detection program for an ambulatory ECG monitor," Biomed. Sci. Instrum., vol. 14, pp. 81-85, 1978.

[12] R. Oweis and, L. Hijazi, "A computer-aided ECG diagnostic tool," computer methods and programs in biomedicine 81, pp. 279-284, 2006.

[13] S. Sachinsingh and N. Netaji Ghandhi, "Pattern analysis of different ECG signal using Pan-Tomkins algorithm ," International Journal on Computer Science and Engineering, vol. 2, no. 7, pp. 2502-2505, 2010.

[14] M. K. Soni, Dr. DipaliBansal, SeemaNayak "Filtering techniques for ECG signal processing", International Journal of Research in Engineering \& Applied Sciences (IJREAS), vol. 2, no.2, pp. 671-679, February 2012.

[15] H. Gurkan, "Compression of ECG signals using variable-length classifed vector sets and wavelet transforms," EURASIP Journal on Advances in Signal Processing, pp. 1-17, 2012.

[16] D. L. Donoho, "Compressed sensing," IEEE Trans. on Information Theory, vol. 52, no. 4, pp. 1289-1306, 2006.

[17] H. Mamaghanian, N. Khaled, D. Atienza, and P. Vandergheynst, "Compressed sensing for real-time energy-efficient ECG compression on wireless body sensor nodes," IEEE Trans. on Biomedical Engineering, vol. 58, pp. 2456-2466, 2011. 
[18] L.F. Polania, R.E. Carrillo, M. Blanco-Velasco, and K.E. Barner, "Compressed sensing based method for ECG compression," in Proceedings, IEEE Int. Conf. on Acoustics, Speech, and Signal Processing, Prague, Czech Republic, ICASSP, , pp. 761-764, May 2011.

[19] A. M. R. Dixon, E. G. Allstot, D. Gangopadhyay, and D. J. Allstot, "Compressed sensing system considerations for ECG and EMG wireless biosensors," IEEE TBCAS, vol. 6, no. 2, pp. 156 -166, April 2012.

[20] L. F. Polania, R. E. Carrillo, M. B. Velasco, and K. E. Barner, "Compressive sensing for ECG signals in the presence of electro myography noise," in 38th Annual Northeast Bioengineering Conference, pp. 295-296, Mar. 2012.

[21] L. F. Polania and K.E. Barner, "A weighted $\ell 1$ minimization algorithm for compressed sensing ECG", 2014 IEEE International Conference on Acoustics Speech and Signal Processing (ICASSP), pp. 1-5, 2014.

[22] M. M. Abo-Zahhad, Aziza I. Hussein, A. M. Mohamed, "Compression of ECG signal based on compressive sensing and the extraction of significant features," Int. J. Communications, Network and System Sciences, vol. 8, pp. 97-117, April 2015.

[23] MIT-BIH 1999, "MIT-BIH arrhythmia database directory," Available online: http://www.physionet.org/physiobank/ database/mitdb/ (Last accessed on 30 September 2015).

[24] M. M. Abo-Zahhad, T. K. Abdel-Hamid, A. M. Mohamed, "Compression of ECG signals based on DWT and exploiting the correlation between ECG signal samples," Inter. J. Communications, Network and System Sciences, vol. 7 no. 1, pp. 53-70, 2014. 
JES, Assiut University, Faculty of Engineering, Vol. 44, No. 2, March 2016, pp. $158-174$

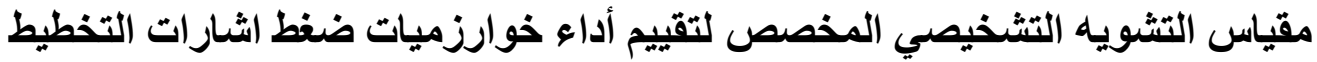

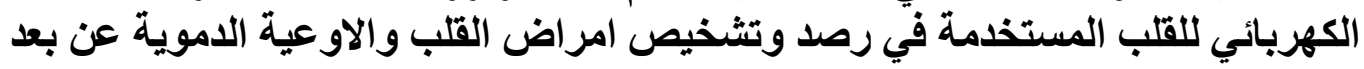

\section{الملخص العربي}

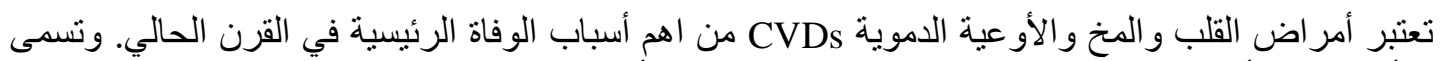

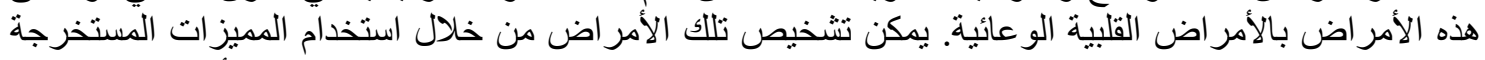

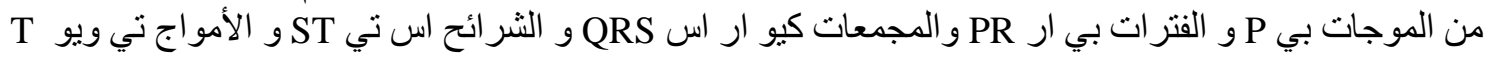

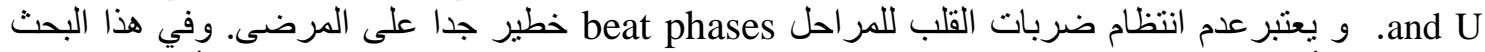

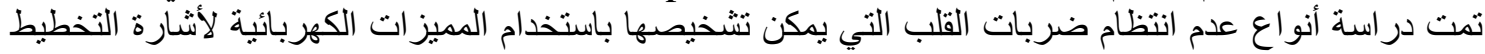

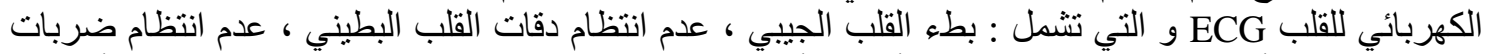

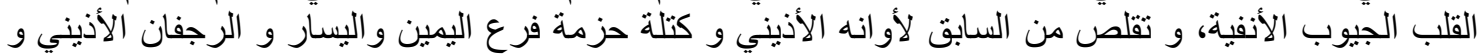

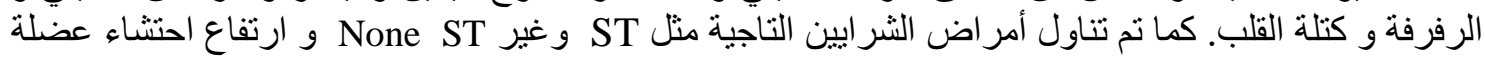

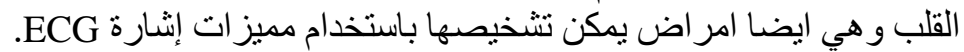

تم في هذه الورقة مناقشة المعايير التقليدية لتقييم جودة الاشار ات المسترجعة من الاشار ات المضغوطة الماته كما تم

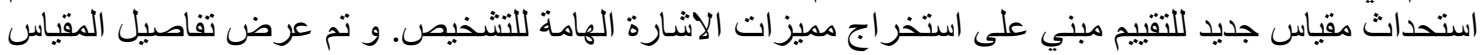

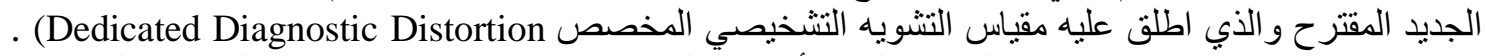

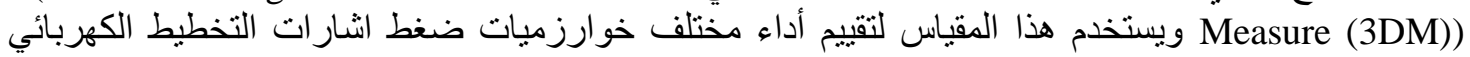

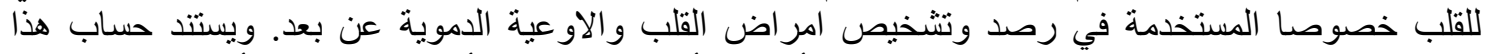

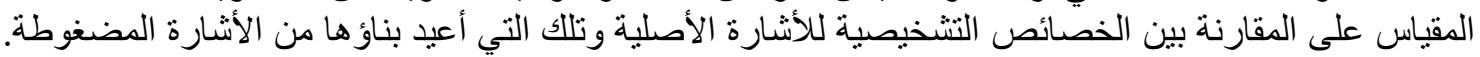

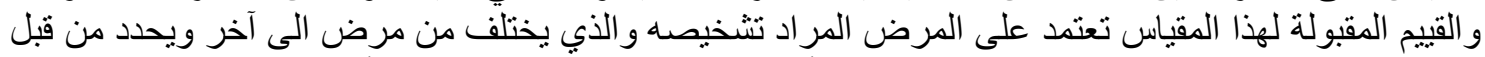

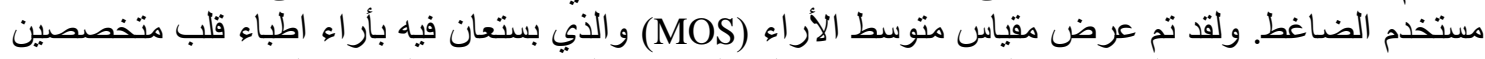

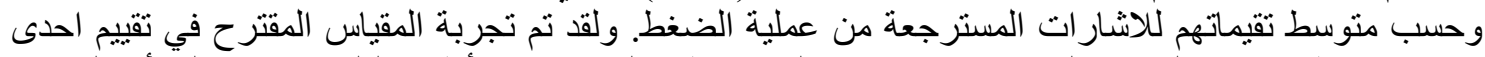

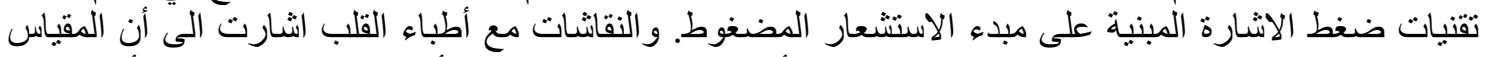

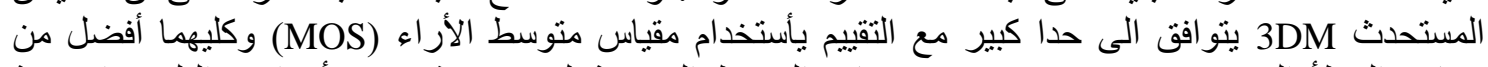

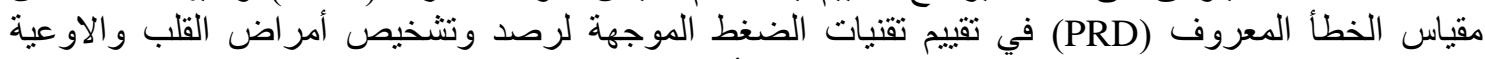

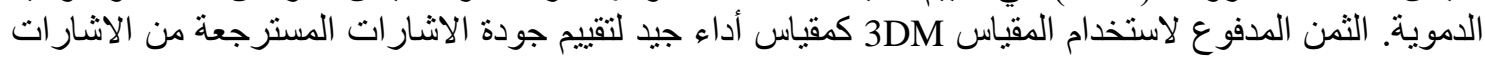

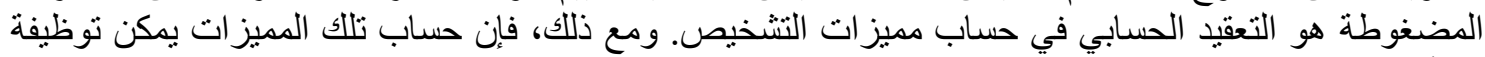

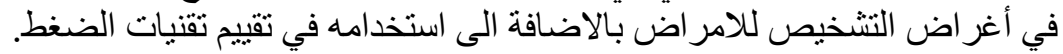

